\title{
Lexical Influences on the Sublexical System during Nonword Spelling
}

\author{
Tami J Patterson and Jocelyn R Folk*
}

Department of Psychology, Kent State University, Kent, Ohio, USA

Received: March 07, 2014; Accepted: April 16, 2014; Published: April 18, 2014

*Corresponding author: Jocelyn R. Folk, Department of Psychology, Kent State University, Kent, Ohio-44242, USA, E-mail: jfolk@kent.edu

\begin{abstract}
We investigated the combined contributions of multiple mechanisms that have been previously indicated to underlie the lexical system's influence on the spelling of unfamiliar words. In two lexical priming studies we presented lists of words and nonwords aurally for participants to spell only the nonwords. Word primes that rhymed with the target nonwords immediately preceded the nonwords (Experiment 1) or there were two intervening items between the prime word and target nonword (Experiment 2). We found that prime words influenced nonword spellings even across intervening items, suggesting that the presentation of a real word may temporarily re-weight sublexical sound-to-spelling correspondences. Importantly, word neighbors were also found to influence nonword spelling, even in the absence of a prime word. Our data provide evidence of the operation of multiple mechanisms that underlie the lexical system's influence on the sublexical system during the spelling of unfamiliar words (or nonwords).
\end{abstract}

Keywords: Spelling; Lexical priming; Lexical-sublexical interaction

\section{Introduction}

There have been many studies indicating that the spellings produced for unfamiliar words can be influenced by the spellings of familiar words [1-5]. However, the mechanisms underlying this influence are not well understood. The goal of the current study was to investigate the underlying mechanisms involved in spelling unfamiliar words, focusing on the multiple ways in which the spellings of familiar words influence the process of spelling unfamiliar words.

Most theories of spelling include two processes involved in the spelling of familiar and unfamiliar words: the lexical and sublexical processes [6-11]. The lexical process is involved in retrieving the spellings of familiar words from a long-term memory store of orthographic information [6,7] (Figure 1). When an unfamiliar word is presented, these theories assume that the sublexical process assembles a spelling based on sound-to-letter correspondences [8]. These correspondences, or phoneme-grapheme (P-G) probabilities, indicate the likelihood of assigning one or more letters for a given sound based on a person's experience with a language.
Once a spelling is activated from either the lexical or sublexical system, subsequent activation occurs at the grapheme level, or graphemic buffer [12], an orthographic working memory process in which the individual graphemes are held active [13] until they are produced in written or spoken output.

While there is evidence that the sublexical system is involved in the spelling of familiar words $[4,11,14,15]$, the focus of the current investigation is on the influence of the lexical system on unfamiliar word spelling. Many studies have reported evidence supporting a role for familiar words influencing the spelling of unfamiliar words using a lexical priming task with unimpaired spellers $[1-5,16,17]$. In this task, participants spell made-up nonwords preceded by real words that are related to the nonwords in some way. Using this methodology, Barry and Seymour [1] found that the spelling of a nonword could be influenced by a rhyming prime word that preceded it, which they interpreted as evidence that spellings activated by real words in the lexical system can influence the spelling of nonwords by the sublexical system.

Folk and Rapp [4] investigated two possible mechanisms responsible for the influence of the lexical system on the sublexical system: dynamic re-weighting of P-G correspondences in the sublexical system by the presentation of a prime word and residual activation of a prime word's letters in the graphemic buffer. Dynamic re-weighting occurs when the P-G correspondences used to spell a word are activated in the sublexical system upon the aural presentation of a familiar word. The resting activation of the P-G correspondences associated with the spelling of the word is temporarily changed (re-weighted) to reflect the spelling in the prime word. When a nonword is subsequently presented for spelling, then the P-G correspondence that was temporarily re-weighted will be more likely to be selected to spell the nonword then if a word prime had not been presented, perhaps because the changes in resting activation cause it to become active more quickly [18]. In contrast, residual activation involves orthographic priming. Upon hearing a real word prime, its letters become active at the grapheme level (Figure 1) and are thus "primed" and more likely to be used in spelling the subsequent nonword. 


\section{Lexical System}

Phonological Representations

Semantic Representations

Orthographic Long-Term Memory

Grapheme Level (Graphemic Buffer)

Phonemes



Conversion
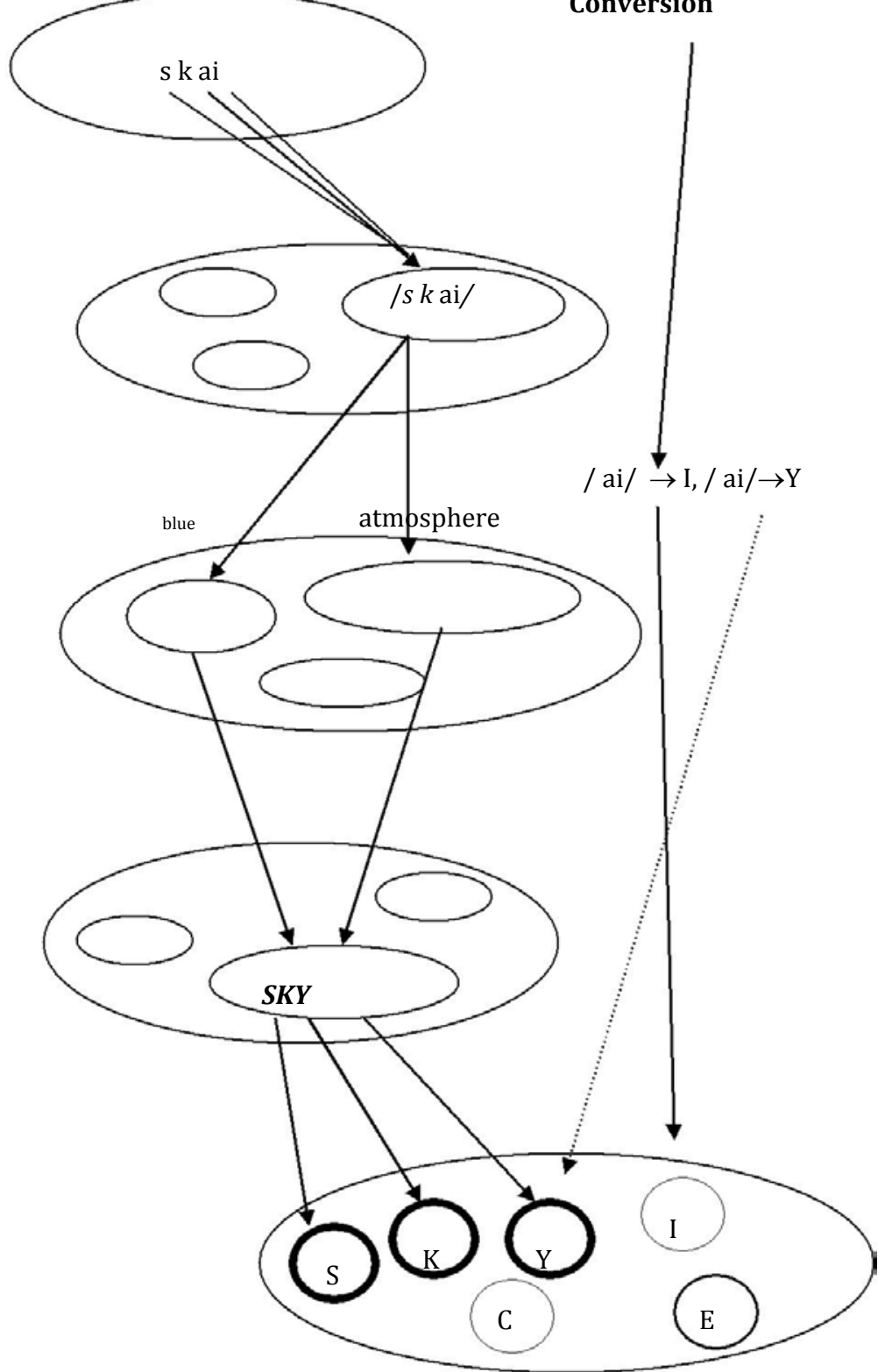

Figure 1: An abbreviated functional architecture of spelling processes, adopted from [4]

Folk and Rapp, in addition to a rhyming prime condition (e.g., touch-/n $\wedge \mathrm{t} \int /$, with target spelling of NOUCH), added an orthographic overlap prime (e.g., couch, $/ \mathrm{n} \wedge \mathrm{t} \int /$, with target spelling of NOUCH). The orthographic condition was included to investigate the extent to which priming was a result of residual activation of a word prime's letters since the prime-nonword pairs overlapped only in orthography. Folk and Rapp found significant priming of a nonword's spelling in both the orthographic prime condition and the rhyming prime condition.

However, priming in the rhyming prime condition was significantly greater than in the orthographic only condition, suggesting that residual activation of a prime word's letters was not the only source of lexical/sublexical interaction in the study. Folk and Rapp proposed that the other mechanism involved is dynamic re-weighting in which the prime word temporarily influences the weightings of P-G correspondences normally used to spell nonwords.

Consistent with Folk and Rapp [4], Perry [18] found that priming effects persist even after two intervening items between the prime word and the target nonword. He interpreted this 
finding as suggesting that lexical priming is not due solely to short-term, residual activation of the letters of prior real words, since the effects lasted even when other stimuli were presented in between the prime word and target nonword, which should eliminate residual activation of the prime word's letters as subsequent words' letters are activated. Perry concluded, similar to Folk and Rapp [4], that changes in the resting activation of P-G rules in the sublexical system are responsible for the priming effects.

Tainturier, et al. [19] found that the activation of phonological word neighbors also influences nonword spellings. They found that French nonwords were spelled more often using lowprobability P-G correspondences when the nonwords had a close phonological neighbor to the target spelling that included the low probability P-G correspondence than for those without a close phonological neighbor. Tainturier et al. concluded that auditory presentation of a nonword activates familiar words in long-term memory that are phonological neighbors of the nonword, including their spellings, which in turn influence how the nonword is spelled. In this way, the lexical system influences the operation of the sublexical system. It is possible that neighbor effects could account for all previous findings as prime words are neighbors of the target nonwords, and their increased activation as a result of being presented could account for priming effects.

\section{Current Study}

Previous work suggests that several mechanisms may underlie the influence of the lexical system on the sublexical system during the spelling of unfamiliar words: 1) dynamic reweighting, which is the temporary re-weighting of P-G rules or correspondences in the sublexical system via the presentation of a real word; 2) residual activation, which is the persisting activation of a prime word's letters at the grapheme level, making them more likely to be selected when a subsequent nonword is spelled; and 3) activation of familiar words that are phonological neighbors of the aurally presented nonword. The spellings of the neighbor words become active and influence the activation of graphemes, which are then more likely to be selected when the nonword is spelled. However, it remains unclear as to whether all three of these mechanism actually contribute to lexical/ sublexical interaction. Specifically, it is possible that previously reported priming effects could be explained, not in terms of priming from residual activation or dynamic re-weighting, but as a result of the activation of word neighbors of the presented nonwords. Additionally, it is unclear, if we find evidence of lexical priming, whether effects of dynamic re-weighting occur over and above effects of residual activation when word neighbor effects are taken into account. Importantly, it is possible that these three possible mechanisms are not mutually exclusive, such that they may each contribute to the lexical/sublexical interaction during nonword spelling and/or interact with one another. To investigate the contribution of these different possible mechanisms to lexical influences on the sublexical system, we employed a lexical priming task, using nonwords as our unfamiliar words.

\section{Experiment 1}

The work of Tainturier et al. [19] suggests that a lexical influence on the operation of the sublexical system may be a result of the activation of the spellings of word neighbors upon the aural presentation of a nonword. Those spellings are more likely to be selected to spell the nonword. Accordingly, what we have been calling priming effects may actually be a result of the increased activation of the prime word, a neighbor of the nonword, making it more likely that the prime word's spelling influences how the nonword is spelled and not other mechanisms. We extended the work of Tainturier et al. [19], who only examined the influence of words with the same target spelling as the nonword (friends), to examine the role that lexical friends and enemies play in nonword spelling. If word neighbors influence the spelling of nonwords, we would expect to see an influence of friends and/or enemies on nonword spelling. Likewise, if friends and/or enemies influence priming effects, we would expect to see an interaction suggesting that the magnitude of priming effects could be influenced by the mean summed frequency of the neighbors of the nonwords.

\section{Method}

Participants: Ninety-six undergraduate students from Kent State University participated in this experiment (48 in the lexical priming condition and 48 in the control condition with 12 per list). Participants received course credit for their participation, and all were native speakers of English.

Materials: A set of 24 prime/nonword target pairs was created. Nonwords were constructed by changing the first phoneme of each prime word. For all lists used in the experiment, each set of 24 target nonword items was divided equally into 4 groups according to all four possible combinations of mean summed frequency of friends (one phoneme differs, and shares same vowel spelling as prime word) and enemies (one phoneme differs, and has a different vowel spelling): "high enemies/high friends" (HE-HF), "high enemies/low friends" (HE-LF) "low enemies/high friends" (LE-HF), and "low enemies/low friends" (LE-LF). Nonwords were considered to be low in friends or enemies if they had a mean summed frequency of less than 50 for friends or enemies respectively, and items were considered to be high if they had a mean summed frequency of 300 or more for friends or enemies [20]. Mean summed frequencies were matched among high and low groups. Also matched were the P-G probabilities of the target vowel spellings [21] for each group to ensure that baseline P-G probabilities did not differentially influence responding. See Table 1 for mean summed frequencies and mean P-G probabilities.

Eight lists were created: 4 for the experimental lexical priming condition and 4 for the control condition. Lists 1-4 for the priming condition contained 24 experimental nonword targets and 24 rhyming prime words, with the prime-nonword pairs in different pseudo-randomized orders in each list. The control lists (5-8) contained nonword targets without rhyming prime words. The prime words were replaced with other real words that neither rhymed with the subsequent nonword nor shared the same vowel sound or spelling. Each list contained 24 
prime-nonword pairs, with each participant hearing a total of 230 items, 182 of which were filler items consisting of both real words (54\%) and made up nonwords (46\%). These filler items served to ensure that prime/nonword pairs were separated by no fewer than six filler items. Each list was divided across two sessions in such a way as to prevent the presentation of duplicate vowel sounds in one session.

\section{Procedure}

An experimenter read lists of words and nonwords aloud to participants at a rate of approximately one item every four seconds. Participants were instructed to put a check on their answer sheet if they heard a real English word, and if they heard a made-up nonword, they were instructed to spell it in a way that seemed reasonable to them. Participants attended two separate sessions exactly one week apart. Each participant in the experimental condition heard only one list containing 24 target nonwords (12 each session) with a rhyming real word immediately preceding each target nonword. Each participant in the control condition heard only one list containing 24 target nonwords (12 each session) with a nonrhyming real word (no prime) immediately preceding each target nonword. Participants were tested in groups or individually. The two testing sessions lasted approximately 20 minutes each.

\section{Results}

The proportion of items for which the target vowel spellings were produced for target nonwords was calculated. For the prime condition, responses were excluded if the prime word was spelled out by the participant or if a checkmark was placed for the nonword instead of the nonword being written, either because the participant thought the stimulus was a real word or misheard it. In the control condition, responses were excluded if a checkmark was placed for the nonword instead of the nonword being written. If participants wrote the real word primes in the prime condition, it is possible that the motor processes might have influenced the subsequent writing of the nonword. Responses excluded for all of these reasons accounted for 7.53\% of the total responses across both prime and control conditions. Data analysis was conducted across participant $\left(F_{1}\right.$ and $\left.t_{1}\right)$ and item $\left(\mathrm{F}_{2}\right.$ and $\left.\mathrm{t}_{2}\right)$ variability.

A 2 (friends: high or low) X 2 (enemies: high or low) $\mathrm{X}$ 2 (condition: prime or control) mixed analysis of variance (ANOVA) was conducted. For the participants analyses, the

Table 1: Mean Summed Frequencies, Mean P-G probability [21] and P-G Range by Condition.

\begin{tabular}{|c|c|c|c|c|}
\hline $\begin{array}{c}\text { Phonological } \\
\text { Neighbors }\end{array}$ & $\begin{array}{c}\text { Mean Sum } \\
\text { Frequency } \\
\text { Enemy }\end{array}$ & $\begin{array}{c}\text { Mean Sum } \\
\text { Frequency } \\
\text { Friend }\end{array}$ & $\begin{array}{c}\text { Mean } \\
\text { P-G } \\
\text { Probability }\end{array}$ & $\begin{array}{c}\text { P-G } \\
\text { Range }\end{array}$ \\
\hline HE-HF & 424 & 389 & 17.68 & $0.45-45.79$ \\
\hline HE-LF & 480 & 36 & 20.55 & $2.65-60.24$ \\
\hline LE-HF & 16 & 361 & 23.17 & $2.43-88.75$ \\
\hline LE-LF & 34 & 35 & 16.35 & $1.20-81.23$ \\
\hline
\end{tabular}

Note: $\mathrm{HE}=$ high enemy, HF = high friend, LE = low enemy, LF = low friend. Word frequency determined using [22] prime condition was a between participants variable while friends and enemies were within-participants variables. For the items analyses, the prime condition was a within-items variable while friends and enemies were between-items variables. The analysis resulted in a significant main effect of condition, $\mathrm{F}_{1}$ $(1,94)=119.75, \mathrm{MSE}=6.03, \mathrm{p}<0.001 ; \mathrm{F}_{2}(1,20)=84.64, \mathrm{MSE}=$ $0.79, \mathrm{p}<0.001$ (Table 2), indicating that participants produced significantly more target spellings in the prime condition $(\mathrm{M}=$ $0.60 \mathrm{SE}=0.016)$ than the control condition $(\mathrm{M}=0.35 \mathrm{SE}=0.016)$, replicating lexical priming effects. The main effect of enemy was significant by participants, $\mathrm{F}_{1}(1,94)=222.93$, MSE $=5.10, \mathrm{p}$ $<0.001$, and marginal by items, $\mathrm{F}_{2}(1,20)=4.22$, MSE $=0.63$, $\mathrm{p}=$ 0.053 , collapsed across prime and control condition, indicating that participants produced more target spellings for nonwords with low enemies $(\mathrm{M}=0.59 \mathrm{SE}=0.014)$ than high enemies $(\mathrm{M}$ $=0.36 \mathrm{SE}=0.013)$. The main effect of friend was significant by participants, $\mathrm{F}_{1}(1,94)=44.28$, MSE $=0.914, \mathrm{p}<0.001$, but not by items, $\mathrm{F}_{2}<1$, collapsed across prime and control. No interactions were significant (Table 2).

\section{Discussion}

Our data indicate that a nonword's phonological neighbors can influence the likelihood of producing the target nonword spelling and that the frequency of those neighbors affects their influence on the operation of the sublexical system. The main effect of enemy indicates that participants are more likely to respond with target spellings of the nonword when the mean summed frequency of word neighbors that are enemies of the nonword is low than when it is high. Importantly, there was no interaction between enemy and condition, indicating that while word neighbors did influence the likelihood of producing a particular nonword spelling, they had no influence on overall priming effects, indicating that lexical priming from a previously presented word occurs. Experiment 2 addresses possible mechanisms of this priming.

\section{Experiment 2}

In Experiment 1 we found priming effects beyond the effects of friends and enemies. Clearly some factor beyond the effect of word neighbors is responsible for a substantial portion of the priming effect. Thus, Experiment 2 was designed to explore priming effects across intervening items to investigate the influence of dynamic re-weighting and residual activation on sublexical processing and the interaction, if any, with lexical influences from word neighbors. Since letter level activation from the presentation of the nonword should be overwritten by the intervening items, any remaining lexical effects on nonword spelling should be a result of the re-weighting process [18].

\section{Method}

Participants: Ninety-six undergraduate students from Kent State University participated in this experiment. Forty-eight of the participants were in the lexical priming condition (12 per list) and forty-eight in the control condition (12 per list). Participants received course credit for their participation. All participants were native English speakers, and none participated 
Table 2: Proportion of trials for which the target nonword spellings were produced in Experiment 1 by participants and by items.

\begin{tabular}{|c|c|c|c|c|c|}
\hline \multicolumn{4}{|c|}{ Phonological Neighbors } \\
\hline Condition & HE-HF & HE-LF & LE-HF & LE-LF & $0.74(0.75)$ \\
\hline Prime & $0.42(0.45)$ & $0.54(0.54)$ & $0.69(0.69)$ & $0.60(0.61)$ \\
\hline Control & $0.18(0.18)$ & $0.18(0.18)$ & $0.40(0.40)$ & $0.52(0.53)$ & $0.35(0.35)$ \\
\hline Priming & $+0.24(+0.27)$ & $+0.25(+0.26)$ & $+0.29(+0.29)$ & $+0.25(+0.26)$ \\
\hline
\end{tabular}

Note: Item means are in parentheses. HE = high enemy, HF = high friend, LE = low enemy, LF = low friend.

in Experiment 1.

Materials: The materials were identical to those in Experiment 1, with the exception that in the experimental lists, prime words were followed by two intervening items which were real words that did not rhyme with or share any vowel sounds or target vowel letters with the target nonword. Each list contained 24 prime-nonword pairs, with a total of 278 items, 230 of which were filler items consisting of both real words (63\%) and nonwords $(37 \%)$. In the control lists, the word prime was replaced with a nonrhyming real word.

\section{Procedure}

The procedure was identical to that used in Experiment 1 , with the exception that the intervening items added to the number of stimuli presented. Participants in the prime condition heard a prime word, then two nonrhyming intervening words, and finally the target nonword, with filler words before the prime words and after the nonwords. Each participant in the control condition heard only one list containing 24 target nonwords, with a nonrhyming real word (no prime) immediately preceding it. Each session lasted approximately 20-25 minutes.

\section{Results}

The data were scored the same as in Experiment 1. Responses excluded accounted for $5.51 \%$ of the total responses across both prime and control conditions. Analyses were conducted for participant $\left(\mathrm{F}_{1}\right.$ and $\left.\mathrm{t}_{1}\right)$ and item $\left(\mathrm{F}_{2}\right.$ and $\left.\mathrm{t}_{2}\right)$ variability.

A 2 (friends: high or low) X 2 (enemies: high or low) X 2 (condition: prime or control) mixed ANOVA was conducted. For the participants analyses, the prime condition was a between participants variable while friends and enemies were withinparticipants variables. For the items analyses, the prime condition was a within-items variable while friends and enemies were between-items variables. The ANOVA yielded a significant main effect of condition, $\mathrm{F}_{1}(1,94)=4.35, \mathrm{MSE}=0.22, \mathrm{p}<0.05 ; \mathrm{F}_{2}(1,20)=$ 11.03, MSE $=0.04, \mathrm{p}<0.01$ (See Table 3 ), indicating significantly more target spellings for the prime condition $(\mathrm{M}=0.43 \mathrm{SE}=0.016)$ than control condition $(\mathrm{M}=0.38 \mathrm{SE}=0.016)$. As in Experiment 1 , the main effect of enemy was significant by participants, $F_{1}(1$, $94)=207.31, \mathrm{MSE}=5.45, \mathrm{p}<0.001$, and approached significance by items, $\mathrm{F}_{2}(1,20)=3.03$, MSE $=0.64, \mathrm{p}=0.097$, indicating more target spellings were produced for nonwords in the low enemies condition $(\mathrm{M}=0.53 \mathrm{SE}=0.015)$ than high enemies $(\mathrm{M}=0.29 \mathrm{SE}=$ 0.013). The main effect of friends was significant by participants, $\mathrm{F}_{1}(1,94)=66.75, \mathrm{MSE}=1.31, \mathrm{p}<0.001$, but not by items, $\mathrm{F}_{2}<1$. As in Experiment 1, there were no significant interactions in the analyses (Table 3).
Cross-Experiment Analysis: In order to examine the extent to which priming effects persisted across two intervening items, a cross-experiment 4-way ANOVA was conducted, with data combined from both Experiments 1 and 2. Results showed a significant main effect of condition, $\mathrm{F}_{1}(1,188)=85.10$, MSE $=4.27$, $\mathrm{p}<0.001, \mathrm{~F}_{2}(1,40)=90.70, \mathrm{MSE}=0.60, \mathrm{p}<0.001$, again suggesting more target spellings for the prime $(\mathrm{M}=0.51 \mathrm{SE}=0.011)$ than control condition $(\mathrm{M}=0.37 \mathrm{SE}=0.011)$. There was a significant main effect of enemy $F_{1}(1,188)=429.07$, MSE $=10.54, p<0.001$; $\mathrm{F}_{2}(1,40)=7.05, \mathrm{MSE}=1.27, \mathrm{p}<0.05$, indicating more target spellings for target words with low enemies $(\mathrm{M}=0.56 \mathrm{SE}=$ $0.010)$ than high enemies $(\mathrm{M}=0.32 \mathrm{SE}=0.009)$. Similarly, there was a main effect of friend by participants only, $\mathrm{F}_{1}(1,188)=$ 109.58, $\mathrm{MSE}=2.21, \mathrm{p}<0.001 ; \mathrm{F}_{2}(1,40)=1.31, \mathrm{MSE}=0.24, \mathrm{p}=.26$, suggesting a trend for more target spellings for low friends (M $=0.49 \mathrm{SE}=0.010)$ than high friends $(\mathrm{M}=0.39 \mathrm{SE}=0.009)$. The main effect of intervening items was significant by participants, $\mathrm{F}_{1}\left(1,188=16.57, \mathrm{MSE}=0.83, \mathrm{p}<0.001\right.$; but not by items, $\mathrm{F}_{2}<1$, suggesting a trend for more target spellings across the prime and control conditions for zero intervening items $(\mathrm{M}=0.47 \mathrm{SE}$ $=0.011)$ than for two intervening items $(\mathrm{M}=0.41 \mathrm{SE}=0.011)$.

Importantly, there was a significant interaction between condition and intervening items, $\mathrm{F}_{1}(1,188)=39.47$, MSE $=1.98, \mathrm{p}$ $<0.001 ; \mathrm{F}_{2}(1,40)=34.84, \mathrm{MSE}=0.23, \mathrm{p}<0.001$, indicating that there was significantly larger priming effects for zero intervening items (Experiment 1$)(\mathrm{M}=+0.25)$ than for two intervening items (Experiment 2) $(\mathrm{M}=+0.05)$. Another way to examine the effects of neighbors is to analyze just the control conditions across experiments. Those analyses led to the same conclusions. There are significantly more target spellings in the LE condition than the HE condition, $\mathrm{t}_{1}(95)=14.84, \mathrm{p}<0.01 ; \mathrm{t}_{2}(23)=2.2, \mathrm{p}<0.05$. The trend for more target spellings for the LF condition than HF is significant only in the analysis by participants, $\mathrm{t}_{1}(95)=8.54, \mathrm{p}$ $<0.01 ; \mathrm{t}_{2}(23)=1.33, \mathrm{p}>0.19$.

\section{Discussion}

The results of Experiment 2 demonstrate that there are multiple mechanisms involved in the lexical system's influence on the sublexical system during the spelling of unfamiliar words (or nonwords), and that previously found priming effects cannot be explained fully as a result of the influence of word neighbors on nonword spelling. We found evidence that the aural presentation of a prime word before a nonword influences the spelling of the nonword, even when there are two intervening words between the prime word and nonword. Neighbor effects did not significantly influence this priming. This is consistent 
Table 3: Proportion of trials for which the target nonword spellings were produced in Experiment 2 by participants and by items.

\begin{tabular}{|c|c|c|c|c|}
\hline \multicolumn{4}{|c|}{ Phonological Neighbors } \\
\hline Condition & HE-HF & HE-LF & LE-HF & LE-LF \\
\hline Prime & $0.24(0.29)$ & $0.35(0.35)$ & $0.52(0.53)$ & $0.61(0.61)$ \\
\hline Control & $0.21(0.21)$ & $0.35(0.35)$ & $0.42(0.42)$ & $0.45(0.45)$ \\
\hline Priming & $+0.03(+0.08)$ & $+0.00(+0.00)$ & $+0.10(+0.11)$ & $+0.55)$ \\
\hline
\end{tabular}

Note: Item means are in parentheses. $\mathrm{HE}=$ high enemy, $\mathrm{HF}=$ high friend, $\mathrm{LE}=$ low enemy, $\mathrm{LF}=$ low friend.

with a mechanism by which the presentation of a prime word temporarily re-weights the P-G correspondences used to spell that prime word. Those correspondences are then more likely to be selected to spell the subsequently presented nonword. However, while priming persisted across two intervening items, it was significantly smaller than when there were no intervening items.

\section{General Discussion}

We used lexical priming methodology to investigate the mechanisms underlying the lexical system's influence on the sublexical system during nonword spelling. Our goal was to better understand the processes involved in spelling unfamiliar words by testing the possible mechanisms of lexical/sublexical interaction against each other. To that end, our data suggest that there are at least two mechanisms by which the spellings of familiar words influence the spelling of unfamiliar words. The first is through the influence of phonological word neighbors. The spelling of a nonword is influenced by the spellings of familiar words that overlap in phonology with the unfamiliar word or nonword. Our data also suggest that dynamic re-weighting can influence the operation of the sublexical system independent of word neighbor effects.

\section{Dynamic Re-weighting}

The current study provides support for the dynamic reweighting proposal of temporary changes to the activation levels of P-G correspondences [4] in the sublexical system due to the aural presentation of a prime word. This is over and above any priming effects that could be attributed to the activation of word neighbors or residual activation. In Experiment 2 when there were two intervening words presented between the prime word and target nonword, we found that the prior presentation of a rhyming word prime influenced the subsequent spelling of a nonword. Significant lexical priming in this condition suggests that there is a mechanism involved in lexical/sublexical interaction in spelling beyond influences from simple letter activation from the word prime at the letter level. Rather, the data indicate that there is something about the presentation of the prime word that increases the likelihood that the target nonword will be spelled similarly to the prime word, even when other words are presented between the prime and target nonword. This is consistent with previous proposals that the presentation of a word results in the activation of the word's constituent P-G correspondences in the sublexical system $[1,4,5,18]$. Perry [18] proposed that the P-G mappings or correspondences have dynamic resting activation levels that are changed temporarily by the presentation of a prime word. These changes to resting activation levels influence how quickly the P-G correspondence information becomes active once the nonword is presented, with the information that is more quickly activated being more likely to be selected to spell the nonword.

However, an examination of Table 2 indicates that this effect was driven by the low enemy condition. The priming effect in the HE-HF condition was only 0.03 and was 0.0 in the HE-LF condition. While the interaction was not significant, these data indicate that dynamic re-weighting effects were attenuated for nonwords with a high mean summed frequency of word neighbors with non-target spellings (i.e., enemies). This suggests that word neighbor influences interacted with the dynamic re-weighting mechanisms, perhaps by activating alternative spellings that competed with the results of dynamic re-weighting.

\section{Word Neighbor Influences}

The influence of word neighbors on the spelling of nonwords was examined by manipulating the mean summed frequencies of a nonword's friends (shared vowel sound and vowel spelling with the target vowel spelling of the nonword) and enemies (shared vowel sound, differed in vowel spelling). In Experiment 1 , we found equivalent priming effects in all four word neighbor groups. Additionally, we found that more target spellings were produced for nonwords having low enemies than those having high enemies across prime and control conditions. While previous work has found an influence of word neighbors on nonword spelling, only the influence of "friends" was examined [19]. We extended these findings to the influence of word enemies and the influence of the frequency of word neighbors ${ }^{1}$. We found that when a nonword has neighbors with a high mean summed frequency that have vowel spellings different than the target or intended vowel spelling of the nonword, participants are less likely to produce the target vowel spelling than when the enemy neighbors have a low mean summed frequency. Since we matched the groups on the P-G probability of the vowel spelling in the nonword, it is not the case that more vowel spellings were produced for low enemies because those spellings were more frequent or probable. Rather, the effect seems to arise as a result of word neighbor characteristics. Importantly, word neighbors did not significantly influence lexical priming effects. That is, there was no evidence to suggest that priming effects are only a result of word neighbor activation, with the prime word

${ }^{1}$ The items analyses for the neighbor effects did not always reach significance when the analyses by participants did. This is most likely the result of the smaller number of items per group (8) and the fact that the items analyses are betweenitems, resulting in a reduction of power to detect differences over the withinparticipant analyses. 
exerting an extra influence because it is a more highly activated neighbor. However, since the prime word is a neighbor (friend) of the target nonword, it may be that the aural presentation of the prime/friend was enough to override the activation of word neighbors based on presentation of the nonword. Nonetheless, the data from E2 indicates that something other than neighbor activation is at play.

The mechanism of word neighbor effects is most likely the following. When a nonword is presented aurally, the phonological representations of similarly sounding words are activated in the phonological input lexicon in the lexical system (Figure 1). Activation from these representations activates their spelling representations in the orthographic output lexicon, which in turn activates the letters involved in their spellings at the letter level (graphemic buffer).

The letters involved in the spellings of the phonological neighbors, in this way, influence which letters are ultimately selected to spell the nonword. The most highly activated letters, determined from the activation of letters involved in neighbor spellings and in activation from the P-G system, will be more likely to be selected to spell the subsequently presented nonword [19]. When those letters are different from those in the target spelling, that would result in fewer target spellings of the nonword.

\section{Residual Activation}

While our data provide no explicit demonstration of residual activation, the significant reduction in the magnitude of the priming from Experiment 1 to Experiment 2 is consistent with a residual activation account of lexical/sublexical interaction. That is, the presentation of a prime word activates its letters within the spelling system, making those letters more likely to be selected to spell a subsequent nonword [4]. However, this activation must be short-lived, or the system would become overloaded with activation as subsequent words are encountered [18]. Thus, the smaller priming in Experiment 2 represented that which was produced by dynamic re-weighting of P-G correspondences alone compared to the larger effects in Experiment 1, which were a result of dynamic re-weighting and residual activation combined.

\section{Conclusions}

Our data are consistent with three mechanisms of lexical influence on the operation of the sublexical system during spelling. First word neighbors can influence how a nonword is spelled, independent of the presentation of a prime word. Second, we found evidence that the presentation of a word activates the P-G correspondences involved its spelling. These correspondences are then temporarily re-weighted, making them more likely to be selected to spell a subsequently presented nonword, and this process does not interact with neighborhood influences. Finally, the reduction in priming with the insertion of intervening items between the prime word and target nonword significantly reduced the size of the priming effects, consistent with the claim that part of the priming in Experiment 1 was a result of the persisting activation of the word primes' letters in the graphemic buffer/orthographic working memory $[4,5,18]$. These data represent some of the first evidence of the interaction of multiple mechanisms that are involved in the influence of the lexical system on the sublexical system during the spelling of unfamiliar words (or nonwords).

\section{References}

1. Barry C, Seymour PHK (1988) Lexical priming and sound-to-spelling contingency effects in nonword spelling. The Quarterly Journal of Experimental Psychology 40(1): 5-40.

2. Campbell R (1983) Writing nonwords to dictation. Brain and Language 19(1): 153-178.

3. Cuetos F (1993) Writing processes in shallow orthography. Reading and Writing 5(1): 17-28.

4. Folk J, Rapp B (2004) Interaction of lexical and sublexical information in spelling: Evidence from nonword priming. Applied Psycholinguistics 25(4): 565-585.

5. Martin D, Barry C (2012) Writing nonsense: the interation between lexical and sublexical knowledge in the priming of nonword spelling. Psychonomic Bulletin and Review 19(4): 691-698.

6. Barry C (1994) Spelling routes (or roots or rutes). In: GDA Brown \& NC Ellis (Eds.), Handbook of spelling: Theory process and intervention. Wiley, Chichester, UK, pp. 27-49.

7. Ellis AW (1982) Spelling and writing (and reading and speaking). In: AW Ellis (Ed.), Normality and Pathology in Cognitive Functions. Academic Press, London,UK.

8. Goodman RA, Caramazza A (1986) Aspects of the spelling process: Evidence from a case of acquired dysgraphia. Language and Cognitive Processes 1(4): 263-296.

9. Houghton G, Zorzi M (2003) Normal and impaired spelling in a connectionist dual-route architecture. Cognitive Neuropsychology 20(2): 115-162

10. Kreiner DS, Gough PB (1990) Two ideas about spelling: Rules and word-specific memory. Journal of Memory and Language 29(1): 103118.

11. Rapp, B, Epstein C, Tainturier MJ (2002) The integration of information across lexical and sublexical processes in spelling. Cognitive Neuropsychology 19(1): 1-29.

12. Caramazza A, Miceli G (1990) The structure of graphemic representations. Cognition, 37: 243-297.

13. Jones AC, Folk JR, Rapp B (2009) All Letters are not Equal: SubGraphemic Texture in Orthographic Working Memory. Journal of Experimental Psychology Learning Memory and Cognition 35(6): 1389-1402.

14. Folk JR, Jones AC (2004) The purpose of lexical/sublexical interaction during spelling: Further evidence from dysgraphia and articulatory suppression. Neurocase 10(1): 65-69.

15. Folk JR, Rapp B, Goldrick M (2002) The interaction of lexical and sublexical information in spelling: What's the point? Cognitive Neuropsychology 19(7): 653-671.

16. Barry C (1992) Interactions between lexical and assembled spelling in English Italian and Welsh. In: C Sterling \& C Robson (Eds.), Psychology spelling and education, Multilingual Matters, Bristol, London, UK, pp. 71-86.

17. Barry C, De Bastiani P (1997) Lexical priming of nonword spelling in the regular orthography of Italian. Reading and Writing 9(5-6): 499517.

18. Perry C (2003) Priming the rules of spelling. The Quarterly Journal of 
Experimental Psychology 56(3): 515-530.

19. Tainturier MJ, Bosse M, Valdois S, Rapp B (2000) Lexical neighborhood effects in pseudoword spelling. $41^{\text {st }}$ Annual Meeting of the Psychonomic Society. New Orleans, LA.

20. Lacruz I, Folk JR (2004) Feedforward and feedback consistency effects for high- and low-frequency words in lexical decision and naming. The Quarterly Journal of Experimental Psychology 57(7): 1261-1284.
21. Hanna PR, Hanna JS, Hodges RR, Rudorf EH Jr (1966) Phonemegrapheme correspondences as cues to spelling improvement. United States Department of Health Education and Welfare, Washington DC, USA.

22. Francis WN, Kucera H 1982 Frequency Analysis of English Usage: Lexicon and Grammar. Houghton Mifflin Company, Boston, USA. 